Research Article

\title{
Efficacy and safety of transdermal diclofenac patch versus intramuscular diclofenac injections in postoperative patients of inguinal hernia
}

\author{
Pranjit Narzaree $^{1 *}$, Mahavir S. Griwan ${ }^{2}$, Janardhan Singh ${ }^{1}$
}

\begin{abstract}
${ }^{1}$ Department of Pharmacology, Pt. B.D.Sharma, PGIMS,

Rohtak, Haryana, India,

${ }^{2}$ Department of Surgery, Pt. B. D. Sharma, PGIMS, Rohtak, Haryana, India
\end{abstract}

Received: 19 January 2016 Accepted: 02 March 2016

\section{*Correspondence to: \\ Dr. Pranjit Narzaree, \\ Email: drpranjit504@yahoo.com}

Copyright: (C) the author(s), publisher and licensee Medip Academy. This is an openaccess article distributed under the terms of the Creative Commons Attribution NonCommercial License, which permits unrestricted noncommercial use, distribution, and reproduction in any medium, provided the original work is properly cited.

\begin{abstract}
Background: The aim of this study was to compare the efficacy and safety of diclofenac transdermal patch with intramuscular diclofenac injections in postoperative patients of inguinal hernia.

Methods: Sixty male participants scheduled for surgery of inguinal hernia were equally randomized into two groups. A transdermal diclofenac patch $100 \mathrm{mg}$ was applied to the participants in the study group 3 hours prior to surgery. In the control group, 2 doses of $75 \mathrm{mg}$ diclofenac sodium injections were given intramuscularly, first dose 2 hours after the surgery and second dose after 12 hours. Pain was assessed postoperatively after an interval of every six hours for 24 hours using visual analogue scale (VAS) and verbal rating scale (VRS). Tramadol $50 \mathrm{mg}$ slow intravenous injection was administered as rescue analgesic. Patients were withdrawn from the study when they asked for rescue analgesia, or when the VAS score was $>5$ and VRS $>2$. Data were analysed using unpaired student's ' $t$ ' test.

Results: Both diclofenac patch and intramuscular injections produced effective analgesia. A gradual increase in pain score was observed for diclofenac patch as compared to intramuscular injections 18 hours after the surgery. Diclofenac patch was well tolerated without any significant side effects.

Conclusions: Transdermal diclofenac patch can be considered as safe and effective method of pain management in postoperative patients of inguinal hernia. But diclofenac intramuscular injection provided better pain relief compared to diclofenac transdermal patch.
\end{abstract}

Keywords: Transdermal, Diclofenac patch, Postoperative analgesia

\section{INTRODUCTION}

The International Association for the Study of Pain (IASP) has defined pain as "an unpleasant sensory and emotional experience associated with actual or potential tissue damage". "Pain is an unpleasant localized sensation caused by the stimulation of sensory nerve endings called as nociceptor which are stimulated by the release of prostaglandins and other chemical mediators released from cells damaged by injury or inflammation. Surgery usually involves damage to the tissues. Although ample evidence indicates that an efficacious postoperative pain treatment reduces morbidity and outcome, recent studies have demonstrated that about $50-70 \%$ of patients experience moderate to severe pain after surgery indicating that post-operative pain remain poorly treated. ${ }^{2}$
The pain of surgery is nociceptive (i.e. it is caused by tissue damage and it is transmitted by normal physiological pathways), acute and for short duration which subsides when the damaged tissue heals. ${ }^{3}$ Adverse effects of untreated postoperative pain include psychological effects (suffering, anxiety, depression, anger), stress response (sympathetic activation, hypertension, tachycardia, increased risk of myocardial infarction, stroke, renal failure), metabolic abnormalities, immobility (chest infection, venous thrombosis, pressure sore, delayed recovery) and development of chronic pain. ${ }^{4}$ The primary reason for trying to control pain after surgery is to relieve patients from suffering, since it is the basic duty of all health care professionals to relieve pain and the most important indication for the treatment of pain after surgery is humanitarian. 
Effective pain control can attenuate the stress response to surgery, reduction in postoperative complications, early recovery and cost savings. Early and aggressive treatment of acute pain may prevent the development of chronic postoperative pain. ${ }^{4}$ For severe pain, the most effective treatment is a strong opioid such as morphine. ${ }^{4} \mathrm{~A}$ combination of different drugs improves the effectiveness of analgesia and by using smaller doses of each drug adverse effects are also reduced. Usually postoperative pain medication involves the use of opioid and nonopioids classes of drugs. The opioid analgesics constitute by far the most important group of medications and remain the mainstay of pain control. ${ }^{8}$ But their efficacy is often limited by the development of tolerance, physical dependence, potential hazards of over sedation and respiratory depression. ${ }^{4}$ Among the non-opioid group of drugs NSAIDs have gained increasing popularity in treating postoperative pain specially diclofenac. Among NSAIDs diclofenac is being used widely for postoperative analgesia by the anesthesiologists. ${ }^{9}$ Their ability to relieve pain and decrease opioid consumption is important as it provides opioid sparing effect. ${ }^{10}$

Diclofenac is available in various forms to treat pain like injectable, topical gel, ophthalmic solution, suppository and transdermal patch to treat pain. ${ }^{11}$ Parenteral or oral administration of diclofenac has its own adverse effects and it also carries boxed warnings related to gastrointestinal and cardiovascular side effects. Therefore, in order to minimize these side effects some alternative has to be introduced which can provide equal effectiveness compared to parenteral and oral, but at the same time which minimizes the unwanted side effects. Diclofenac transdermal patch is a newly introduced delivery system for postoperative pain management. It bypasses the first pass metabolism offers painless administration, increased bioavailability, maintenance of constant and prolonged drug level, reduced frequency of dosing, minimization of inter and intra patient variability, self-administration and easy termination of medication, leading to better patient compliance. Diclofenac diethylamine is reportedly used for topical application. ${ }^{12}$

Several researchers have demonstrated the effectiveness of transdermal diclofenac patch in various pain management studies. Pradel, et al used diclofenac patch for acute traumatic blunt soft tissue injuries and they found that patch was effective and well tolerated. ${ }^{13}$ Efficacy of transdermal diclofenac patch was demonstrated in patients with osteoarthritis. ${ }^{14}$ Comparable analgesic effect of diclofenac patch and intramuscular injection was also demonstrated by Alessandri, et al in patients undergoing laparoscopic benign gynecologic surgery. ${ }^{15}$ Krishna and Nataraj demonstrated that intraoperative application of transdermal diclofenac patch was as effective as intramuscular diclofenac injection. ${ }^{16}$

Recent reviews have also suggested that topical NSAIDs are effective, especially against the pain related to acute soft tissue injuries and chronic rheumatic disorders but the evidence has been described as insufficient by the British Society for Rheumatology. ${ }^{17-19}$ On the other hand, the American College of Rheumatology has recommended the use of topical analgesics in patients who do not respond to acetaminophen and wish to avoid systemic therapy. ${ }^{20}$ Despite of the fact that transdermal patch have an approved status for clinical utility, there is scepticism that topical NSAIDs have any action other than as a rubefacient hence more randomised and placebo controlled studies were demanded. ${ }^{21,22}$ Most of the studies are done on painful conditions related to closed type of wounds. Various literatures have been searched but very few studies have discussed regarding the efficacy and safety of transdermal diclofenac patch for pain management related to open surgery. As this innovative drug delivery mechanism provides several advantages. Therefore, present study was undertaken in patients undergoing surgery for inguinal hernia under spinal anaesthesia with the objective to evaluate and compare the efficacy and safety of transdermal diclofenac patch with intramuscular diclofenac injection for postoperative analgesia.

\section{METHODS}

\section{Study design}

This was a prospective, open label comparative study. The present study was conducted in department of pharmacology and surgery at Pt. B. D. Sharma PGIMS Rohtak, approved by the Institutional review board.

\section{Patient population}

Sixty male patients $18-60$ years of age scheduled for surgery of inguinal hernia and eligible as per inclusion and exclusion criteria were enrolled for this study. Enrolled patients were divided into two equal groups (30 each) and allocated to receive one of the 2 different treatments in an open fashion and subjected for clinical assessment.

\section{Inclusion criteria}

Male patients 18 to 60 years of age scheduled for inguinal hernia surgery.

\section{Exclusion criteria}

1. Patients with history of asthma, hypersensitivity reaction to any component of diclofenac.

2. Previous history of bypass heart surgery, myocardial infarction.

3. Patients with severe hepatic and renal disorders and other co-morbid conditions. Diabetes, Tuberculosis, HIV.

4. Gastrointestinal tract related problems (bleeding, perforation and ulcer). 
5. Skin diseases (eczema, fungal infection, inflammation burn wound).

6. Patient with history of alcohol abuse, blood disorders (porphyria bleeding or clotting disorders), and vascular disorders.

\section{Drug interventions}

Detailed patient's histories including general physical and systemic examinations were done. Patients were explained about the procedure, purpose, risks of this study and the postoperative interpretation of visual analogue scale (VAS) and verbal rating scale (VRS). A patient's information sheet was provided to each of the eligible candidate for this study and thereafter an informed written consent was taken from all the participants for this study. Sixty enrolled cases for this study were divided randomly (using computer generated random numbers) into two groups of 30 each.

Group 1 patients received first dose of $75 \mathrm{mg}$ diclofenac sodium $(3 \mathrm{ml})$ intramuscular injection 2 hours after the surgery and second dose was repeated 12 hours after the 1 st dose.

Group 2 patients received transdermal diclofenac diethylamine patch (Nu patch $100 \mathrm{mg}$ ), 3 hours before the surgery and the patch was placed on the upper arm near deltoid region. Transdermal diclofenac patch was applied for a period of 24 hours.

On the previous day of the surgery patients were subjected to pre-anaesthetic check-up, investigation reports were checked accordingly. Vitals were monitored preoperatively and postoperatively. In postoperative ward, assessment for intensity of pain was done by visual analogue scale (VAS) and verbal rating scale (VRS) for every six hours for a total duration of 24 hours. If a patient represented with VAS score $>5$ and VRS $>2$, necessitating urgent analgesia then in such situation there was a provision for rescue treatment, injection tramadol hydrochloride (50mg slow i.v. injection) followed by withdrawal of the candidate from the study.

\section{Clinical assessments}

Clinical assessments were done before and after operation (after drug administration).

\section{End points for postoperative analgesia}

\section{Primary end points}

Measurement for the intensity of pain postoperatively at $6,12,18 \& 24$ hours by

1. Visual analogue scale $(\mathrm{VAS})^{23}$ of $0-10$ points.

2. Verbal rating scale (VRS) ${ }^{24}$ of scores 0- 4 .

\section{Secondary end point}

Number of times rescue analgesic required.

\section{Safety assessment}

Patients were assessed for the presence of any kind of side effects. If in case major toxicity to diclofenac injection or patch occurs in patients, necessitating discontinuation of treatment then the subjects are meant to be withdrawn from the study by giving appropriate treatment.

\section{Statistical analysis}

Results were analysed statistically using student's ' $\mathrm{t}$ ' test (unpaired). $\mathrm{P}$ value of less than 0.05 was to be considered as significant.

\section{RESULTS}

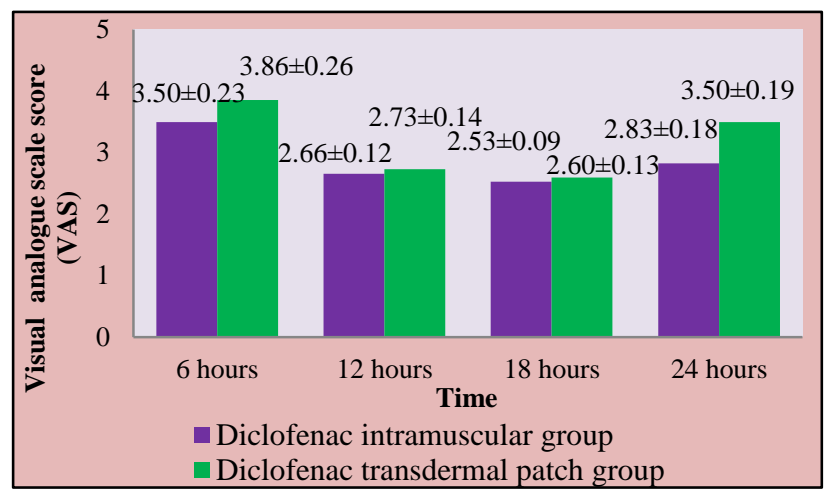

Figure 1: VAS score at different time intervals for diclofenac i.m injection versus transdermal patch group in postoperative patients of inguinal hernia.

The demographic data with respect to age was comparable between the 2 groups. The mean age group for diclofenac intramuscular injection group and diclofenac patch group was 46.9 and 47.4 respectively. Pain was assessed postoperatively using VAS and VRS score in both the groups. Mean VAS score in patients of group 2 (diclofenac patch group) was 3.86 \pm 0.26 , $2.73 \pm 0.14,2.60 \pm 0.13$ and $3.50 \pm 0.19$ after $6,12,18$ and 24 hours respectively. On the other hand mean VAS score in group 1 patients (diclofenac intramuscular injection group) was $3.50 \pm 0.23,2.66 \pm 0.12,2.53 \pm 0.09$ and $2.83 \pm 0.18$ after $6,12,18$ and 24 hours respectively. Mean VAS score for both the groups were at the level of mild intensity throughout the observation period. However, the VAS score obtained at 24 hours showed statistically significant difference between the two groups. The mean pain score as assessed by VRS score in diclofenac transdermal patch group i.e. for group 2 was $1.60 \pm 0.09,1.06 \pm 0.46,1.03 \pm 0.03$ and $1.40 \pm 0.09$ at 6,12 , 18 and 24 hours respectively. While mean VRS score for group 1 i.e. diclofenac intramuscular group was $1.53 \pm 0.09,1.03 \pm 0.03,1.00 \pm 0.00$ and $1.13 \pm 0.06$ at 6,12 , 
18 and 24 hours respectively. Similarly the analgesic effect reflected by verbal rating scale (VRS) scores were also at mild level of intensity, but at 24 hours of postoperative period significant statistical difference was observed in the mean scores between the two groups. Results are shown in Figure 1 and 2.

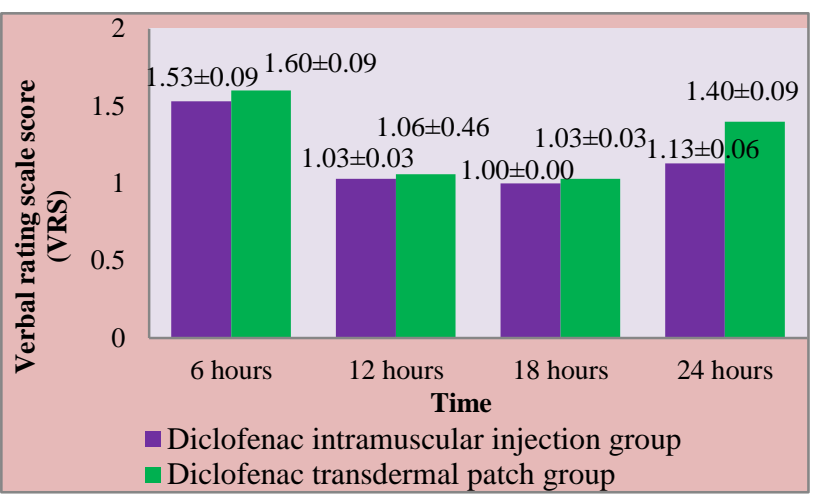

Figure 2: VRS score at different time intervals for diclofenac i.m injection versus transdermal patch group in postoperative patients of inguinal hernia.

\section{DISCUSSION}

Postoperative pain remains an important area of research for introduction of new modalities of treatment with enhanced effectiveness at the same time with lesser side effects. An efficacious postoperative pain management reduces patient morbidity and improves the patient's outcome. Recent studies have demonstrated that about $50-70 \%$ of patients experience moderate to severe pain after surgery indicating that the postoperative pain remains poorly treated. ${ }^{2}$ The possible reason could be distinct mechanism of incisional nociception, compared to other painful conditions. Another reason might be lack of knowledge in depth regarding the pathophysiology and neuropharmacology of postoperative pain. Postoperative incisional pain is unique and common form of acute pain, because surgery usually involves damage to the tissue. Postoperative pain medication is usually managed by opioid and non-opioid group of drugs. Opioid analgesic constitutes by far the most important group of drugs and remains the mainstay of postoperative pain control. ${ }^{8}$ Although opioids are effective postoperative analgesics, but their efficacy is often limited by the development of tolerance, physical dependence, potential hazards of over sedation and respiratory depression. ${ }^{4}$ Among the nonopioid group of drugs NSAIDs have gained increasing popularity in treating postoperative pain specially diclofenac. However, parenteral or oral administration of diclofenac has its own adverse effects and it also carries boxed warnings. Current recommendations for postoperative pain management consist of combination of analgesics i.e. multimodel analgesia. Among NSAIDs diclofenac is being used widely for postoperative analgesia by the anesthesiologists. Alone or in combination with opioids they decrease postoperative pain. And in some cases NSAIDs reduces the requirements of opioids as much as by one third which constitutes the opioids sparing effect of NSAIDs. Topical application of NSAIDs has been reported to be effective in decreasing both acute and chronic pain. ${ }^{25}$ Transdermal patch which is a new innovation in the drug delivery system offers many advantages such as painless administration of drug, increased bioavailability, maintenance of constant and prolonged drug level, reduced frequency of dosing, minimization of inter and intra patient variability, easy self-administration and easy termination of medication, leading to better patient compliance. $^{12-14}$ It also offers a good option for those patients who are unable to administer drug orally. As it bypasses first pass metabolism in the liver, a transdermal drug administration also overcomes concerns regarding drugs that are poorly absorbed in the gastrointestinal tract.

As safety is concerned, topical NSAIDs have potential advantage over oral NSAIDs. Naedal \& Brown demonstrated that because of low systemic concentrations, topical NSAIDs have a reduced risk of upper gastrointestinal complications, such as gastric ulcers and other gastrointestinal symptoms like dyspepsia. $^{26}$ But an important concern with regard to transdermal drugs delivery is its prolonged duration of onset and offset compared to intravenous or intramuscular medication which can be used as when needed to control pain. ${ }^{27}$ Therefore, use of a transdermal patch requires proper planning and careful timing schedule before application in order to get effective results. In patients scheduled for surgery these agents are applied prior to the surgical intervention i.e. in anticipation to pain and not after the patient experiences pain, this is due to the long duration of its onset.

In our study, pain was assessed postoperatively after every 6 hours for a period of 24 hours duration using VAS and VRS score. A VAS score of more than 5 and VRS score of more than 2 were considered need for additional analgesics or rescue analgesics. Injection tramadol 50mg i.v was made available as rescue analgesic. However administration of diclofenac i.m injection in the postoperative patients of inguinal hernia resulted in significant analgesic effect throughout the postoperative period i.e. at $6,12,18$ and 24 hours. In terms of diclofenac transdermal patch the analgesic effect was comparable with diclofenac intramuscular injections and the intensity of pain was continuously maintained at mild level throughout the observation period. At the postoperative period of first 6 hours, patients in both the groups reported pain however it was below moderate level of intensity. In the subsequent postoperative periods of 12 and 18 hours the intensity of pain was maintained at mild level in both the groups. Although the intensity of pain was at mild level during the first 6 hours of postoperative period, but the scores for both the groups are higher than the scores that are obtained at end of 12 and 18 hours. This increase in pain scores at the first 6 hours in both the groups could be due to the rapid sensitization of the nociceptors by the inflammatory mediators due to surgical intervention. When the pain 
scores obtained from the initial 6 hours were compared between the 2 groups it was observed that the pain score was a bit higher for diclofenac transdermal patch compared to diclofenac intramuscular injections, this could be due to low plasma concentration of diclofenac provided by diclofenac transdermal patch. Hence, difference in pain scores was observed during the initial 6 hours of post-operative period. At 24 hours duration patients from both the groups reported pain of mild intensity. But the pain score was more in case of diclofenac transdermal patch treated group in comparison to diclofenac intramuscular treated group. In both the groups the pain was adequately controlled and the pain scores were below moderate level of intensity. Figure 1 and 2 shows gradual increase in pain score for diclofenac transdermal patch after 18 hours of postoperative period compared to patients receiving intramuscular diclofenac. This indicated that single dose $100 \mathrm{mg}$ transdermal diclofenac patch has effectively managed pain till 24 hours of postoperative period. But as the duration increased towards 24 hours its effectiveness gradually decreased, which is reflected by mean VAS and VRS scores. Increase in pain scores for patients treated with transdermal diclofenac patch could be due to reduced concentration of diclofenac, as the diclofenac transdermal patch was applied once in 24 hours comparative to 12 hourly dose of $75 \mathrm{mg}$ diclofenac intramuscular injection. This observations have indicated that in order to get maximum analgesic effect with transdermal patch, either the frequency of application should be increased i.e. patch should be applied 12 hourly or the dose of the drug should be increased in the transdermal patch. However, in both the conditions in comparison to intramuscular diclofenac injection low systemic concentration of diclofenac is anticipated as well as its related toxicities. Similar results were also observed when pain was assessed by using VRS score.

Our results have similarities with the study results that were observed by other researchers. Alessandri \& colleagues found that transdermal diclofenac patch administration is like a valid help to standard analgesic treatment in postoperative pain control and could also reduce the period of hospitalization of patients who undergo laparoscopic benign gynecologic surgery. ${ }^{28}$ Funk L. \& Colleagues concluded that diclofenac patches provides significantly better pain relief compared to tablets in the postoperative period following arthroscopic shoulder surgery. ${ }^{29}$ Pradel et al used a diclofenac patch for acute traumatic blunt soft tissue injuries, and they found that the diclofenac patch was effective and well tolerated. ${ }^{13}$ Transdermal patch is found to be effective and safe for the treatment of sports related blunt impacted injuries. Other studies have also reported effectiveness of diclofenac patch in osteoarthritis. ${ }^{14}$

Results of the present study have demonstrated the analgesic effect of diclofenac transdermal patch in postoperative patients of inguinal hernia. Effectiveness of diclofenac in postoperative pain indicated that some inflammatory components may also be involved particularly in the process of wound healing.

Safety analyses revealed no apparent serious adverse effects. The diclofenac patch was well tolerated with 3 minor cases of nausea and epigastric discomfort. In any of the participants skin reactions have not been observed, comparative to other studies where application site skin reactions were often reported. In diclofenac intramuscular injection treated group one case of burning micturation associated with mild pain while passing urine was observed, another case of mild chest pain and other single cases of nausea and cough have been reported. During the study, poor adhesiveness of transdermal patch was also observed the patch would loosen and peel off during sleep. Therefore frequent checking was needed. A better alternative to this problem could be application of the patch over the immovable part of the body such as chest or abdomen rather than on arm.

Our study results revealed that when applied 3 hours before surgery a diclofenac 100mg transdermal patch had effectively reduced postoperative pain in patients of inguinal hernia. And based on our observations, with proper time schedule application of transdermal diclofenac patch in postoperative patients of inguinal hernia can be considered as safe and effective method of pain management. But in comparison to diclofenac intramuscular injection, although the scores obtained from both the groups were under the mild level of intensity throughout the observational period, but the VAS and VRS scores obtained from the diclofenac patch group showed differences in scores, particularly at 24 hours where significant difference was observed both clinically and statistically. From the observations it can be concluded that intramuscular diclofenac injection provided better pain relief compared to diclofenac patch.

The aim of our efforts was to ensure that pain control receives high priority in the health care system with the introduction of drugs that provides maximum effectiveness, minimum unwanted effects, cost effective and with better patient compliance. Specific efforts and developmental programs are needed to generate expertise in pain management keeping in mind that pain is subjective and individual variability in pain perception should be taken into account.

\section{CONCLUSION}

When applied 3 hours before surgery a diclofenac 100mg transdermal patch had effectively reduced postoperative pain in patients of inguinal hernia with minimum side effects therefore it can be considered as safe and effective method of pain management. But diclofenac intramuscular injection provided better pain relief compared to diclofenac transdermal patch.

\section{Funding: No funding sources}

Conflict of interest: None declared

Ethical approval: The study was approved by the Institutional Ethics Committee 


\section{REFERENCES}

1. Merskey H, Albefessard DC, Bonica J. J Pain termsA list with definitions and notes usage. Pain 1979;6:249-52.

2. Esther M, Pogatzki-Zahn EM, Zahn PK, Brennen TJ. Postoperative pain - Clinical implication of basic research. J Best Pract Res Clin Anesthsiol. 2007;21(1):3-13.

3. Schimer BD. Pain management and conscious sedation. In: Mattox BE, Sabiston Text Book of Surgery. The biological basis of modern surgical practice. 16th ed. Philadelphia: WB Saunders. 2001:283-91.

4. Cuschieri A, Grace PA, Dargi A, Borley M, Rowley DI. Pain relief. In: Clinical Surgery. 2nd ed. Massachusetts: Blackwell Publishing Ltd. 2003:1018.

5. Siddall PJ, Cousins MJ. Neurobiology of pain . J Int Anesth Clin. 1997;35(2):1-26.

6. Sorkin IS, Wallase MS. Acute pain mechanism. J Surg Clin North Am 1999;79(2):213-29.

7. Kim PS, Tobias M, Ferrante FM. Acute pain management of the surgical patient. J Contemp Surg. 1999;55(2):84-95.

8. Yaksh TL, Wallace MS. Opioids, analgesia and pain management. In: Brunton LL, Chabner BA, Knollmann BC, Goodman \& Gilman's The pharmacological basis of therapeutics. 12th ed. Newyork: McGraw Hill. 2011:481-525.

9. Place Galan C, Mujika JA, Elosegui JL, Irureta I, Bollo E, Esteban E, et al. A singlebrectal diclofenac dose reduces post haemorrhoidectomy urine retention. Cir. Esp. 2008;83(6):301-5.

10. Rang H, Dale M, Ritter J. Rang and Dale's Pharmacology, 6th edition. London, England: Churchill Livingstone. 2007.

11. Shang AB, Gan TJ. Optimising postoperative pain management in the ambulatory patient. Drugs. 2003;63(9):855-67.

12. Kriwet K, Muller Goymann CC. Binary diclofenac diethylamine - water system, Micelles, Vesicles and lyotropic liquid crystals. J Eur Pharm Biopharm. 1993;39(6):234-8.

13. Predel HG, Koll R, Pabst H. Diclofenac patch for topical treatment of acute impact injuries: A randomized double blind placebo controlled multicenter study. J Br Sports Med. 2004;38(3):31823.

14. Bruhimann P, Micheal BA. Topical diclofenac patch in patients with knee osteoarthritis: A randomized double blind controlled clinical trial. J Clin Exp Rheumatol. 2003;21(2):193-8.

15. Alessandri F, Lijol D, Mistrangela E. Topical diclofenac patch for postoperative wound pain in laporoscopic gynecologic surgery: A randomized study. J Minim Invas Gynecol. 2006;13(3):195-200.

16. Krishna R, Nataraj MS. Efficacy of a single dose transdermal diclofenac patch as pre-emptive postoperative analgesia: A comparison with intramuscular diclofenac. J South Afr Anaesth Analg. 2012;18:194-7.

17. Moore RA, Tramer MR, Carroll D, Wiffers PJ Mc Quay HJ. Quantitative systematic review of topically applied non-steroidal anti-inflammatory drugs. BMJ. 1998;316:333-8.

18. Rosenstein ED. Topical agents in the treatment of rheumatic disorders. Rheum Dis Clin North Am. 1999;25899-918.

19. Scott DL, Shipley M, Dawson A, Edwards S, Symmons DPM, Woolf AD. The clinical management of rheumatoid arthritis and osteoarthritis: strategies for improving clinical effectiveness. Br J Rheumatol. 1998;37:546-554.

20. American College of Rheumatology Subcommittee on Osteoarthritis Guidelines, Recommendations for the medical management of osteoarthritis of the hip and knee: 2000 update. Arthritis Rheum. 2000;43:1905-15.

21. Bateman DN, Kennedy JG. Non-steroidal antiinflammatory drugs and elderly patients. The medicine may be worse than the disease. BMJ. 1995;310:817-8.

22. Ogilvie-Harris DJ, Gilbart M. Treatment modalities for soft tissue injuries of the ankle: a critical review. Clin J Sport Med. 1995;5:175-86.

23. Gould D. Visual Analogue Scale (VAS). Journal of Clinical Nursing. 2001;10:697-706.

24. Cork R, Isaac I, Elsharydah A, Saleemi S, Zavisca F, Alexander L. A Comparison Of The Verbal Rating Scale And The Visual Analog Scale For Pain Assessment. The Internet Journal of Anesthesiology. 2003;8(1):1.

25. Agarwal A, Dhiraaj S, Kumar A, Singhal V, Singh U. Evaluation of a diclofenac ransdermal patch for the attenuation of venous cannulation pain: a prospective, randomized, double blind, placebocontrolled study. Anaes. 2006;61:360-2.

26. Naedal J, Brown K. NSAID-associated adverse effects and acid control aids to prevent them: a review of current treatment options.Drug Safety. 2006;29(2):119-32.

27. Hitesh B. A prolonged release parenteral drug delivery system. Int Jour Pharmaceu Sci Rev and Res. 2010;3:1-11.

28. Alessandri A, Lijoi D, Mistrangelo E, Nicoletti A, Crosa M, Ragni N. Topical diclofenac patch for postoperative wound pain in laporoscopic gynecologic surgery: A randomized study. Journal of minimally invasive Gynecology. 2006;13(3):195200.

29. Funk L, Umaar R, Molajo A. Diclofenac patches for postoperative shoulder pain. Int J Shoulder Surg. 2008;2:47-8.

Cite this article as: Narzaree P, Griwan MS, Singh J.

Efficacy and safety of transdermal diclofenac patch versus intramuscular diclofenac injections in postoperative patients of inguinal hernia. Int J Basic Clin Pharmacol 2016;5:447-52. 